\title{
Hot wire chemical vapor deposition: limits and opportunities of protecting the tungsten catalyzer from silicide with a cavity
}

\author{
P.A. Frigeri ${ }^{1}$, O. Nos ${ }^{1,2}$, S. Bengoechea ${ }^{1}$, C. Frevert ${ }^{1}$, J.M. Asensi ${ }^{1}$, J. Bertomeu ${ }^{1}$.
}

1. Dept. de Física Aplicada i Òptica, Universitat de Barcelona

Barcelona-08028, Spain, Ph: +34934039221, fax: +34934039219

2. Ecotècnia (ALSTOM Group)

Email of corresponding author: oriol_nos@ub.edu

\begin{abstract}
Hot Wire Chemical Vapor Deposition (HW-CVD) is one of the most promising techniques for depositing the intrinsic microcrystalline silicon layer for the production of micro-morph solar cells. However, the silicide formation at the colder ends of the tungsten wire drastically reduces the lifetime of the catalyzer, thus limiting its industrial exploitation. A simple but interesting strategy to decrease the silicide formation is to hide the electrical contacts of the catalyzer in a long narrow cavity which reduces the probability of the silane molecules to reach the colder ends of the wire. In this paper, the working mechanism of the cavity is elucidated. Measurements of the thickness profile of the silicon deposited in the internal walls of the cavity have been compared with those predicted using a simple diffusion model based on the assumption of Knudsen flow. A lifetime study of the
\end{abstract}


protected and unprotected wires has been carried out. The different mechanisms which determine the deterioration of the catalyzer have been identified and discussed.

\section{Keywords}

$\mu \mathrm{c}-\mathrm{Si}$; Solar cell; Hot Wire Chemical Vapor Deposition; Silicide; Lifetime; Tungsten

\section{Introduction}

Intrinsic microcrystalline silicon ( $\mu \mathrm{c}-\mathrm{Si}: \mathrm{H})$ is being more and more studied since its implementation as active layer in micro-morph solar cells [1]. HW-CVD has the potentiality of depositing ( $\mu \mathrm{c}-\mathrm{Si}: \mathrm{H})$ at very high deposition rate [2,3] using a technology which, in principle, is easy to scale up to very large areas [4,5].

However, its industrial exploitation is strongly limited due to the short lifetime of the catalyzers. The silane $\left(\mathrm{SiH}_{4}\right)$, used as source gas, induces the formation of silicide $\left(\mathrm{WSi}_{\mathrm{x}}\right)$ at the surface of the tungsten (W) wire employed as catalyzer. Moreover, the radical desorption kinetics from the filament profoundly change with silicide formation [6,7]. The aging of the catalyzer implies a low reproducibility of the deposited material quality. Likely, to obtain microcrystalline silicon, silane is strongly diluted with hydrogen $\left(\mathrm{H}_{2}\right)$, which partially removes the silicide by reduction. Silicide formation is also minimized by using high filament temperature: $1850^{\circ} \mathrm{C}$ [8]. Nevertheless, all this beneficial effects are not sufficient to prevent the formation of silicides at the colder ends of the filament. 
A simple but interesting solution to overcome this effect is to hide the electrical contacts of the catalyzer in a long narrow cavity. Frequently, this solution is combined with the introduction of $\mathrm{H}_{2}$ or of an inert gas in the cavity which avoids the colder ends entering in contact with $\mathrm{SiH}_{4}[4,9]$. This paper shows how the cavity alone is able to protect efficiently the colder ends of the catalyzer.

Firstly, the profile of the silicon film deposited on the internal walls of the cavity has been measured for two different partial pressures of $\mathrm{SiH}_{4}$. These results have been compared with those predicted by a model used to clarify the functioning of the cavity.

Secondly, filament lifetime tests have been performed using conditions with higher silane concentration than those typically adopted for the deposition of microcrystalline silicon. These conditions permit to accelerate the silicidation process, thus reducing the time needed for the tests. The lifetime of a catalyzer protected with a cavity has been compared with such of an unprotected catalyzer.

\section{Experimental Details}

The experiments for this research were carried out in a single high vacuum chamber fitted with a hot wire assembly. A spring system allows keeping a tungsten wire of $0.2 \mathrm{~mm}$ of diameter straight, at a constant tension of about $100 \mathrm{~N} / \mathrm{mm}^{2}$, despite of thermal expansion. Two stainless steel cylindrical cavities with an internal diameter of $4 \mathrm{~mm}$ and a length of 90 
$\mathrm{mm}$ were assembled at the colder ends of the wire. The temperature of the cavity never exceeded $200^{\circ} \mathrm{C}$.

In order to measure the thicknesses of the films deposited on the walls of the cavity, one of the wire holders was modified in such a way to be able to fix a glass substrate on the internal wall of the cylindrical cavity. The thickness profiles along the axis of the cavity were measured with a profilometer. A wire of the same length as the cavity $(90 \mathrm{~mm})$ was used for these tests. However, preliminary studies using longer wires did not show any substantial difference in the thickness profiles of the deposited Si layers.

Two different deposition conditions were chosen: Normal pressure deposition $\left(\Phi_{\mathrm{SiH} 4}=10\right.$ sccm, $\Phi_{\mathrm{H} 2}=90$ sccm, $\left.\mathrm{P}=5 \times 10^{-2} \mathrm{mbar}, \mathrm{P}_{\mathrm{SiH} 4}=\mathrm{P} \cdot \Phi_{\mathrm{SiH} 4} /\left(\Phi_{\mathrm{H} 2}+\Phi_{\mathrm{SiH} 4}\right)=5 \times 10^{-3} \mathrm{mbar}\right)$ and low pressure deposition $\left(\Phi_{\mathrm{SiH} 4}=2 \mathrm{sccm}, \Phi_{\mathrm{H} 2}=0 \mathrm{sccm}, \mathrm{P}=\mathrm{P}_{\mathrm{SiH} 4}=2 \times 10^{-4} \mathrm{mbar}\right)$. In both cases the wire temperature was set at $1850{ }^{\circ} \mathrm{C}$, estimating the temperature by measures of the resistance of the wire.

The lifetime of $34 \mathrm{~cm}$ length catalyzer wires was tested with and without the protection of a cavity under the following fixed deposition conditions: $\Phi_{\mathrm{SiH} 4}=10 \mathrm{sccm}, \Phi_{\mathrm{H} 2}=90 \mathrm{sccm}$, $\mathrm{P}=5 \times 10^{-2}$ mbar, $\mathrm{P}_{\mathrm{SiH} 4}=\mathrm{P} . \Phi_{\mathrm{SiH} 4} /\left(\Phi_{\mathrm{H} 2}+\Phi_{\mathrm{SiH} 4}\right)=5 \times 10^{-3}, \mathrm{~T}_{\mathrm{f}}=1850{ }^{\circ} \mathrm{C}$. Morphological studies and chemical analysis of the tested wires were carried out using SEM (Jeol JSM-840) equipped with an x-ray microanalysis system (INCA Energy 250, Oxford Instruments).

\section{Results and discussion}




\subsection{Cavity mechanism}

Fig. 1 shows the normalized thickness profiles of the Si layers deposited on the internal walls of the cavity along its axis. Tests have been carried out at two different silane partial

pressures of $\mathrm{P}_{\mathrm{SiH} 4}=5 \times 10^{-3}$ mbar, and $\mathrm{P}_{\mathrm{SiH} 4}=2 \times 10^{-4}$ mbar. In both cases, the results have been fitted with a hyperbolic cosine curve of the form:

$$
\frac{d(x)}{d_{o}}=\frac{\cosh ((x-L) / \lambda)}{\cosh (L / \lambda)}
$$

where $\lambda$ is the length which characterizes the decay of the thickness profile along the cavity axis $\mathrm{x}$, and $L$ is the length of the cavity.

It is a reasonable approximation to suppose that the thickness profiles are directly proportional to the amount of silane dissociated by the filament at the same position $t \cdot R \cdot n(x)$, where $t$ is the deposition time, $R$ the dissociation rate and $n(x)$ the linear silane density. This means that $d(x)$ and $n(x)$ are formally solutions of the same problem. Since the length of the cavity $L$ is much bigger than its radius r, i.e $L>>r$, Eq.1 follows as solution of the one dimensional diffusion problem for $n(x)$, which reads

$$
D \frac{d^{2} n(x)}{d x^{2}}-R \cdot n(x)=0
$$


being $D$ the diffusion constant. The boundary conditions are: $n(0)=n_{0}$, i.e the cavity is open in $x=0$, and $d n(L) / d x=0$, i.e. the cavity is closed at its end. The $\mathrm{SiH}_{4}$ concentration $n(x)$ and the thickness profile decay exponentially with a characteristic length scale $\lambda=\sqrt{D / R}$. The mean free path of silane molecules $\lambda_{\mathrm{SiH} 4}$ is given by [10]

$$
\lambda_{\mathrm{SiH}_{4}}=k T /\left(\sqrt{2} \cdot P_{\mathrm{SiH}_{4}} \cdot \pi \cdot d^{2}{ }_{\mathrm{SiH}_{4}}\right)
$$

where the diameter of the molecules has been estimated to be $\mathrm{d}_{\mathrm{SiH} 4}=0.232 \mathrm{~nm}$ [11] and the gas temperature to be around $\mathrm{T}=600 \mathrm{~K}$, which is a mean value between the wire temperature and the temperature that reaches the inner cavity wall due to filament radiation. Considering these parameter values and the pressures previously mentioned, the mean free path is $\lambda_{\mathrm{SiH} 4}>>1 \mathrm{~cm}$. Since the mean free path of silane molecules is much bigger than the diameter of the cavity, the system is subject to a Knudsen flow. Hence, according to the kinetic theory of gases, the diffusion constant for a pipe is given by [10]

$$
D=2 r \cdot \nabla / 3
$$

where $r$ is the radius of the cavity and $v$ the mean speed of the $\mathrm{SiH}_{4}$ molecules. The dissociation rate is given by the number of molecules striking the wire per unit length and unit time multiplied by the probability of the $\mathrm{SiH}_{4}$ dissociation $\gamma$, which gives

$$
R \cdot n(x)=r_{f i l} \cdot \gamma /\left(2 \cdot r^{2}\right) \cdot \nabla \cdot n(x)
$$


where $r_{\text {fil }}$ is the radius of the tungsten filament. The theoretical estimation of $\lambda$ with $\mathrm{r}_{\mathrm{fil}}=0.1 \mathrm{~mm}, \mathrm{r}=2 \mathrm{~mm}$ and $\gamma=0.4[12]$ gives

$$
\lambda=\sqrt{\frac{4}{3} \frac{r^{3}}{r_{\text {fil }} \cdot \gamma}}=16.3 \mathrm{~mm} \text { (6). }
$$

The characteristic length scale $\lambda$ resulting from the fitting of the experimental data is about $16 \mathrm{~mm}$ for $\mathrm{P}_{\mathrm{SiH} 4}=5 \times 10^{-3} \mathrm{mbar}$ and $12 \mathrm{~mm}$ for $\mathrm{P}_{\mathrm{SiH} 4}=2 \times 10^{-4} \mathrm{mbar}$. Both values are in good agreement with the theoretical prediction. The silane concentration decays exponentially in the cavity. The decay factor is fully determined by the two radius of the system and by the dissociation probability of the catalyzer. The cavity offers the opportunity to protect the cold ends of the catalyzer from silicide reducing exponentially the concentration of silane along its axis if its length $L>>\lambda$. However, the effective length of the cavity can be drastically shorter than the geometrical one if its end is not perfectly sealed.

\subsection{Lifetime test}

Fig.2 shows the studied regions of the filaments protected with a cavity or not. The typical process of breakage of a non protected filament takes place at a colder region near the electrical contact, (region 2 in Fig.2), where the temperature is too low for the catalytic reaction but high enough to permit silicidation. In this region, silicon difusses rapidly into the wire core forming $\mathrm{WSi}_{2}$ composites as deduced from the $\mathrm{Si}: \mathrm{W}$ ratios shown in Table 1. 
The filament becomes fragile, tends to crack, and finally breaks. Comparing Fig.3(b) with Fig.3(c) as well as the different Energy Dispersive Spectroscopy (EDS) results of Table 1, it's clear that the effect of this process is strongly reduced if the filament is protected by a cavity. Moving closer to the electrical contact, the silicide formation is heavily reduced due to the low temperature of the wire, so a pure silicon layer grows on its surface, see Fig.3(a). In fact, for large deposition times, at this cold region, silicides are finally formed under the silicon layer of the surface, see Fig.4(a). For short deposition times, the central part of the catalyzer remains unaffected with superficial silicon contents below $5 \%$.

The unprotected filament broke in about 1 hour, while the protected wire did not break after more than 13 hours of uninterrupted deposition. Furthermore, the experiment was carried out with higher silane concentration than those usually used for microcrystalline Si growth. Lifetime of the wires is expected to increase if microcrystalline silicon deposition conditions were chosen, i.e $\sim 5 \% \mathrm{SiH}_{4}$ dilution instead of $10 \%$. In any case, the cavity does not avoid the silicidation at the cold ends, not even at the contact region, see Fig. 4, but it drastically slows down the process, thus reducing the effects on the mechanical stability of the wire. Looking at the EDS results in Table 1 for this wire, it is clear that silicidation is present in every one of the differenciated regions, including the central one. Moreover, once the silicidation process of each region is advanced, a layer of pure silicon begins to grow on its surface. As the wire does not break in the first hours of deposition, the main limitation of the cavity protection system is the degradation of the central part of the wire, see Fig.4(c), which loses its catalytic capacity due to the slow but progressive silicide formation. 
For a better wire protection it is necessary to implement solutions acting on the catalytic stability of the central part of the catalyzer. The most popular methods to regenerate the catalyzer involve annealing treatments [13-15]. However, these methods extend the life of the catalyzer only if executed for every deposition run. The whole procedure is time expensive, thus being not very interesting for industrial implementation. In particular, it is not suitable for uninterrupted deposition processes, like roll-to-roll production systems. In any case, these procedures are standard methods used in all HW-CVD laboratory systems involved in silicon deposition. Another possibility is to coat the tungsten wire with a thin carbide layer [16]. This strategy seems to protect efficiently the catalyzer from silicide formation but it is not clear if the carbide layer affects its catalytic efficiency to decompose the silane molecules.

\section{Conclusions}

Catalyzer lifetime extension from 1 to 13 hours has been achieved by assembling a cylindrical cavity at the cold ends of a $0.2 \mathrm{~mm}$ diameter tungsten filament. Deposition conditions involved higher silane concentrations than those used for microcrystalline $\mathrm{Si}$ growth. Catalyzer lifetime is expected to be further increased if microcrystalline silicon deposition conditions are chosen to carry out the tests. The protective mechanism follows from the combination of a small diffusion constant with a large silane dissociation rate in the cavity, leading to exponential silane concentration decay along the cavity axis. The cavity efficiently protects the catalyzer cold ends if its length $L$ is much bigger than the 
characteristic length $\lambda$ (Eq.3). The main limitation of this approach is the impossibility to avoid the slow but progressive silicidation of the catalyzer central part, which finally looses its catalytic properties. Up to now, there is no thorough solution to solve this last problem.

\section{References}

1. H. Keppner, J. Meier, P. Torres, D. Fischer, A. Shah, Appl. Phys.A 69 (1999) 169177.

2. J. Cifre. J. Bertomeu, J. Puigdollers, M. Polo, J. Andreu, A. Lloret, Appl. Phys.A: Matee. Sci. Process. 59 (1994) 645

3 R.E.I. Schropp, Y. Xu, E. Iwaniczko, G.A. Zaharias, A.H. Mahan, in: J.D. Cohen, J.R. Abelson, H. Matsumura, J. Robertson (Eds.), Amorphous and Heterogeneous Silicon-Based helds at the 2002 MRS Spring Meeting, San Francisco, USA, April 25, 2002, Material Research Society Symposium Proceeding 715 (2002) A26(3.1).

4. K. Ishibashi, M. Karasawa, G. Xu, N. Yokokawa, I. Manabu, A. Masuda, H. Matsumura, Thin Solid Films 430 (2003) 58-62.

5. A. Ledermann, U. Weber, C. Mukherjee, B. Schröeder, Thin Solid Films 395 (2001) 61-65. 
6 J.K. Holt, M. Swiatek, D.G. Goodwin, H.A. Atwater, in: J.D. Cohen, J.R. Abelson, H. Matsumura, J. Robertson (Eds.), Amorphous and Heterogeneous Silicon-Based helds at the 2002 MRS Spring Meeting, San Francisco, USA, April 2-5, 2002, Material Research Society Symposium Proceeding 715 (2002) A23(3.1).

7. A.H. Mahan, A. Mason, B.P. Nelson, A.C. Gallagher, Mater. Res. Soc. Symp. Proc. 609 (2001) A6.6.1.

8. E. Iwaniczko, A.H. Mahan, B. Yan, L.N. Gedvilas, D.L. Williamson, B.P. Nelson, in:J.R. Abelson, G. Ganguly, H. Matsumura, J. Robertson, E.A. Schiff (Eds.), Amorphous and Nanocrystaline Silicon-Based Films helds at the 2003 MRS Spring Meeting, San Francisco, USA, April 22-25, 2003, Material Research Society Symposium Proceeding 762 (2003) 387-392.

9. $\quad$ K. Honda, O. Keisuke, H. Matsumura, Thin Solid Films 516 (2008) 826-828.

10. R.D. Present, Kinetic Theory of Gases, McGraw-Hill, New York, 1958.

11. J.V. Sali, S.B. Patil, S.R. Jadkar, M.G. Takwale, Thin Solid Films 395 (2001) 66-70.

12. N. Honda, A. Masuda, H. Matsumara, J. Non-Cryst. Solids 266 (2000) 100-104.

13. C.H.M. van der Werf, P.A.T.T. van Veenendaal, M.K. van Veen, A.J. Hardeman, M.Y.S. Rusche, J.K. Rath, R.E.I. Schropp, Thin Solid Films 430 (2003) 46. 
14. D. Knoesen, C. Arendse, S. Halindintwali, T. Muller, Thin Solid Films 516 (2008) $822-825$.

15. D. Hrunski , B. Schroeder, Thin Solid Films 516 (2008) 814 - 817.

16. K. Honda, K. Ohdaira, H. Matsumura, Thin Solid Films 516 (2008) 826 - 828.

\section{Acknowledgements}

This work has been supported by the EU through the FLEXCELLENCE project (contract 019948) and the Spanish Governement through the project CLASICO (ENE2007-67742C04-03) and PSE-MICROSIL08. One of the authors was financially supported through the Stefano Franscini fonds.

\section{Figure captions}

Fig.1 Normalized thickness profile of the Si layers deposited on the internal walls of the cavity along its axis. The squares and the triangles show the data obtained for $\mathrm{P}_{\mathrm{SiH} 4}=5 \times 10^{-3}$ mbar, and $\mathrm{P}_{\mathrm{SiH} 4}=2 \times 10^{-4}$ mbar respectively. The continuous and the dotted lines have been obtained fitting the data with Eq. 1.

Fig.2 Studied regions of the tungsten wires tested: (1) at the electrical contact; (2) cold ends; (3) central region. SEM images were taken from these regions of the wires.

Fig.3 Unprotected wire after 77 min of test: (a) cross section of region 1, and (b) region 2. 
Protected wire after 77 min of test: (c) region 2 (see Fig.2).

Fig.4 Protected wire after 13 h of test: (a) cross section of region 1, (b) region 2, and (c) region 3 (see Fig.2).

Table1 EDS analysis of Si:W atomic ratio from the different regions described in Fig.1. *Si:W ratio of 65.1:34.9 found on the external core zone. 
Table 1

\begin{tabular}{clccc} 
& & $\begin{array}{c}\text { Unprotected } \\
\text { wire after } \\
77 \mathrm{~min}\end{array}$ & $\begin{array}{c}\text { Protected } \\
\text { wire after } \\
77 \mathrm{~min}\end{array}$ & $\begin{array}{c}\text { Protected } \\
\text { wire after } \\
13 \mathrm{~h}\end{array}$ \\
\hline \hline \multirow{2}{*}{ Region } & central core & $0: 100$ & $0: 100$ & $0: 100 *$ \\
& surface & $100: 0$ & $65.2: 34.8$ & $98.7: 1.3$ \\
\hline \multirow{2}{*}{2} & central core & $63.3: 36.7$ & $0: 100$ & $65.4: 34.6$ \\
& surface & $66.2: 33.8$ & $45.9: 54.1$ & $94.3: 5.7$ \\
\hline \multirow{2}{*}{3} & central core & $0: 100$ & $0: 100$ & $68.4: 31.6$ \\
& surface & $3.7: 96.3$ & $3.7: 96.3$ & $99.8: 0.2$
\end{tabular}


Figure1

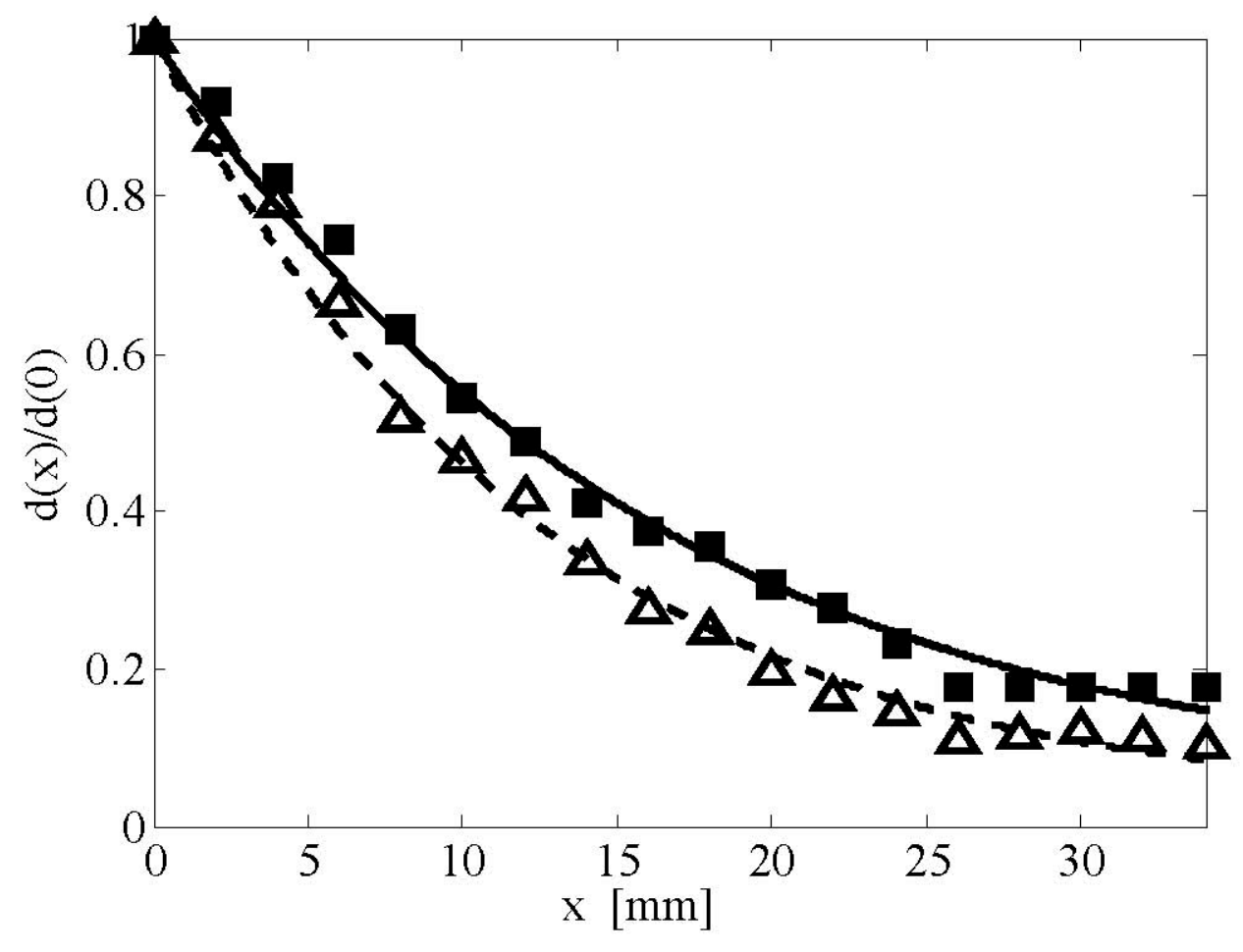


Figure 2

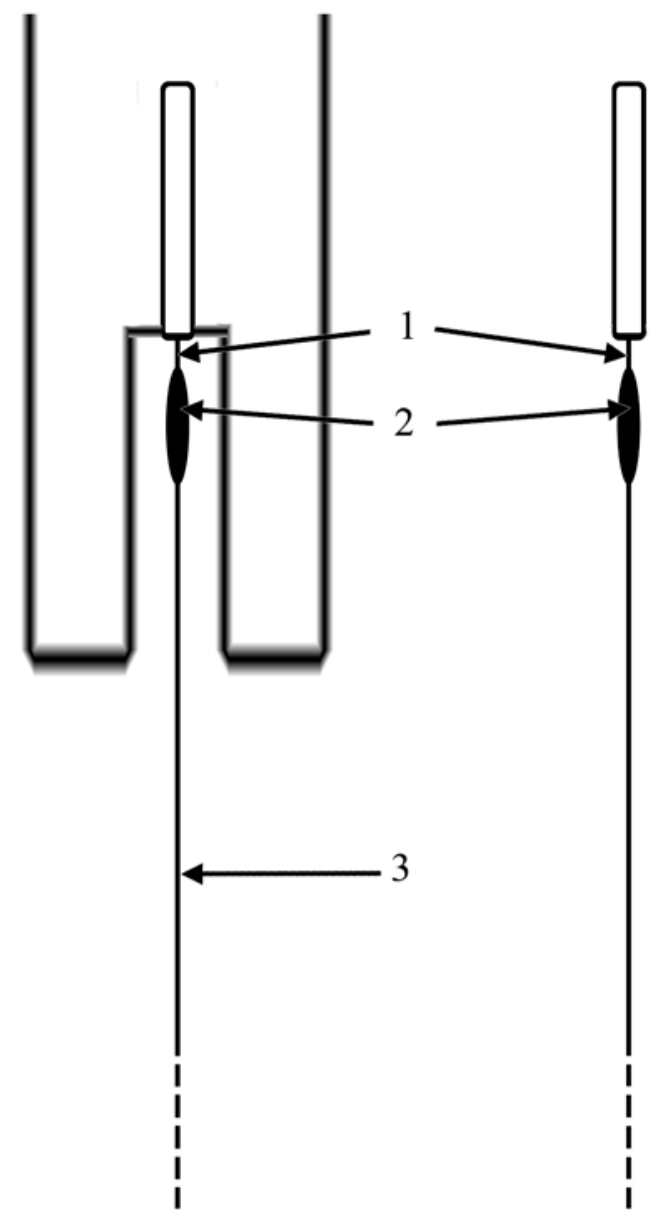


Figure 3

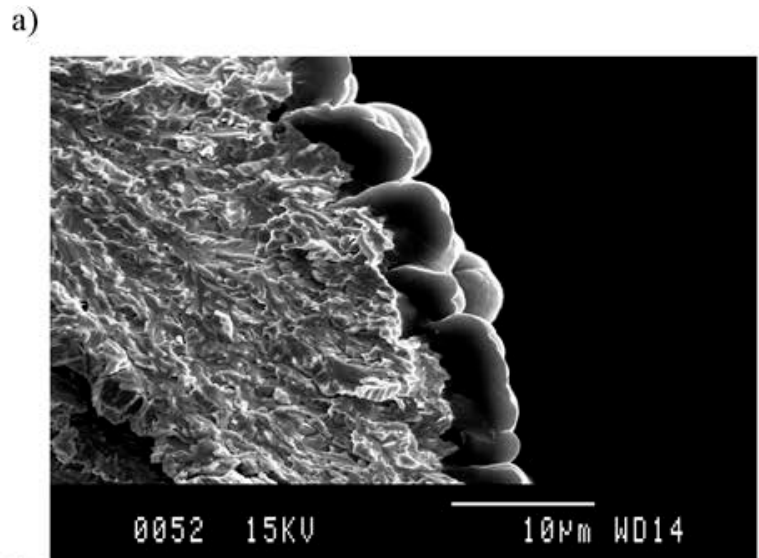

b)

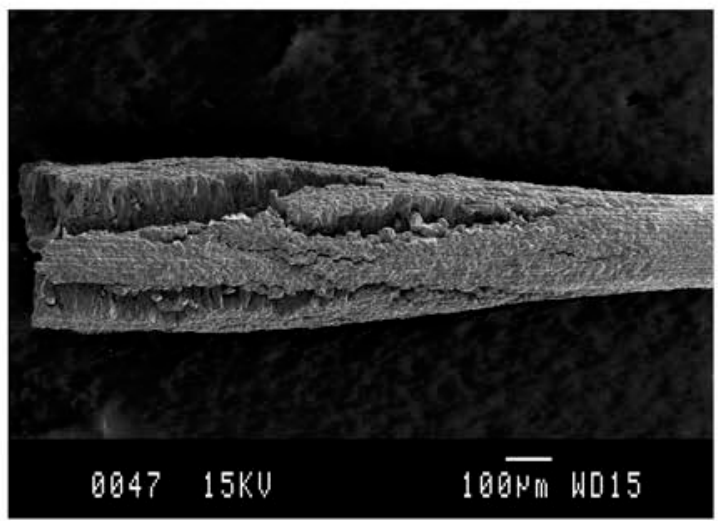

c)

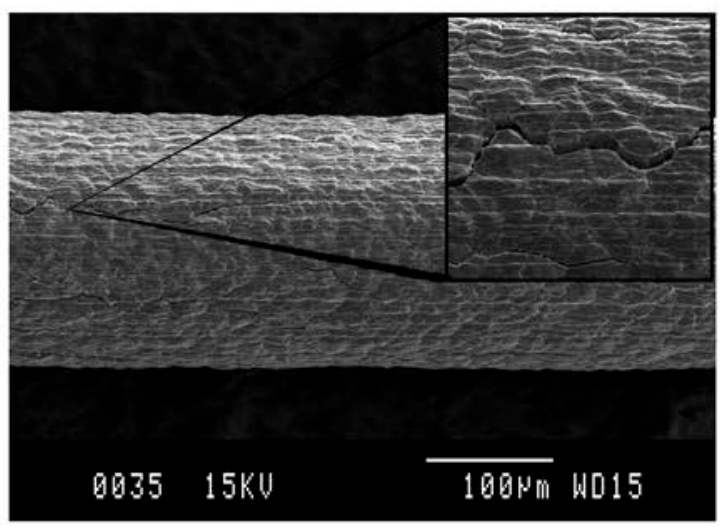


Figure 4

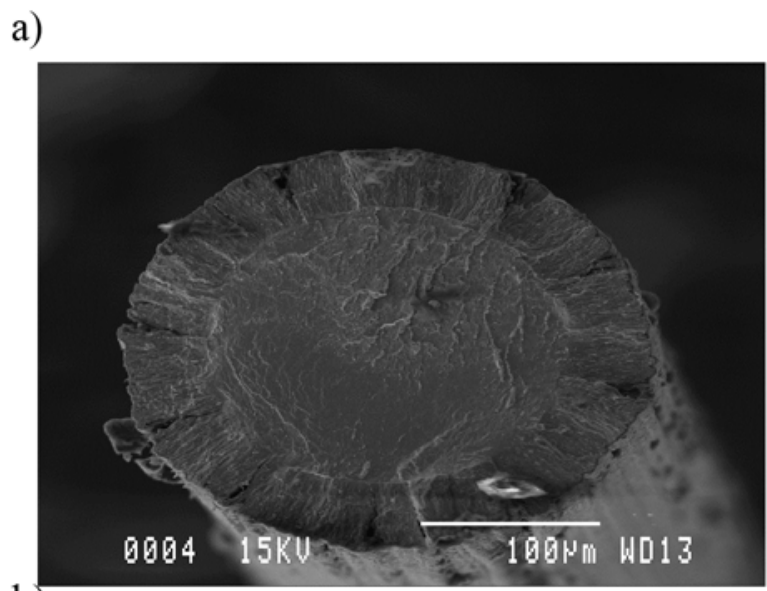

b)

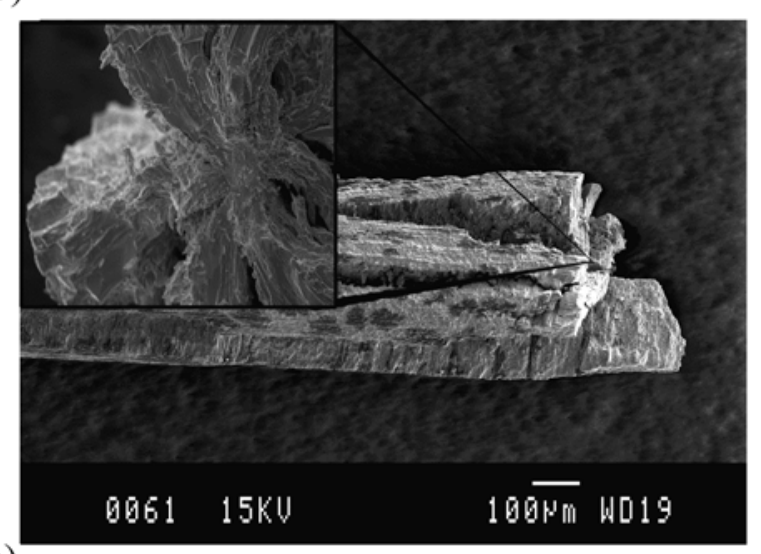

c)

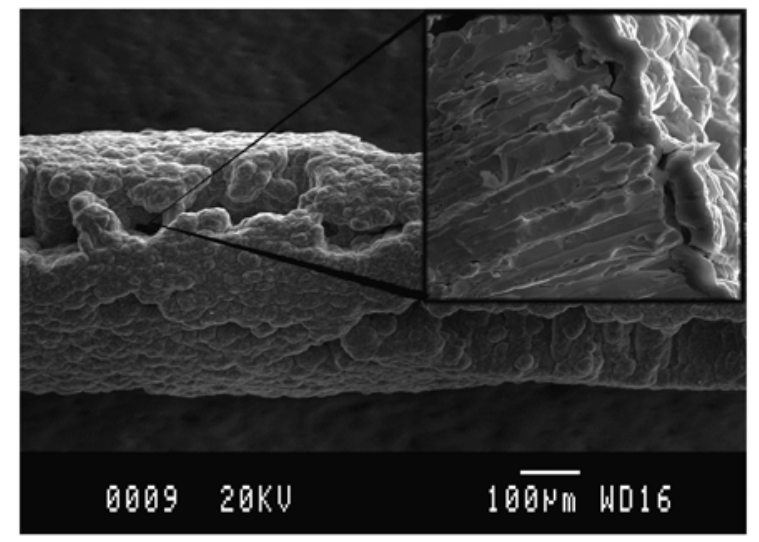

